\title{
Plasmonic Refractive Index Sensing Based on Interference in Disordered Composite Films
}

\author{
Boris Okorn ${ }^{1}$, Vesna Janicki ${ }^{1}$, Tonica Bončina ${ }^{2}$, Franc Zupanič², Jordi Sancho-Parramon ${ }^{1,}$ \\ ${ }^{1}$ Ruđer Bošković Institute, Bijenička cesta 54, 10000 Zagreb, Croatia \\ ${ }^{2}$ University of Maribor, Faculty of Mechanical Engineering, Smetanova 17, 2000 Maribor, Slovenia
}

Key words: plasmonic sensors, thin films, interference, nanocomposites, metal nanoparticles

*Corresponding author: e-mail jsancho@irb.hr

The strong dispersion in the effective optical constants of plasmonic nanocomposite films can be used to generate interference effects that are highly sensitive to changes in the dielectric environment of particles. Numerical simulations show that reflectance measurements on metal nanoparticles systems close to a metallic substrate have larger sensitivity to changes of surrounding refractive index than standard transmittance measurements of the same system when placed on a transparent substrate. The scheme is particularly advantageous in case of disordered nanoparticles systems, where the difference between reflectance and transmittance-based sensing approaches is enhanced due to local field fluctuations that modify the effective optical constants dispersion. Exceptionally to most plasmonics applications, in the present case a disordered system provides larger sensitivity and figure of merit than its ordered counterpart. The key concepts suggested from numerical calculations are verified by fabrication of metal island films coated with different dielectric layers, that confirm the superiority of the reflectance-based sensing scheme. Overall, the present approach takes profit from disorder to improve refractive index sensitivity in metal nanoparticle systems that can be produced with industrially appealing techniques. Thus, the proposed scheme may be valuable for broad implementation of low-cost and highly efficient plasmonic sensors.

Copyright line will be provided by the publisher

The last two decades have witnessed a remarkable development of plasmonics due to its large application potential in multiple areas ranging from telecommunications to medical diagnoses and therapies [1]. However, many of the most exciting plasmonic applications have been demonstrated only at laboratory level and their widespread use is still far from being implemented. The main reason is that many of these applications demand an accurate control of nanostructure fabrication and often require the use of techniques that are inadequate for industrial processes. Thus, there is a quest for the design of novel schemes that can enable plasmonic applications to be realized using inexpensive approaches.

Sensing was among the first areas in which the application potential of plasmonics was demonstrated. The characteristics of surface plasmon oscillations strongly depend on the refractive index of the medium surrounding metal nanostructures, regardless of whether they are metal films [2] or nanoparticles [3]. Electromagnetic field enhancement and hence plasmonic sensing can be increased, for instance, using waveguide modes [4] or magnetic resonances [5]. Amid many schemes, plasmonic-based chemical and biological sensing has been demonstrated using metal island films (MIFs), i.e., near two-dimensional random distributions of metal nanoclusters that are formed during the first stages of metal deposition on dielectric surfaces [6]. Such structures can be fabricated by industriallysuitable standard thin film fabrication techniques and their plasmonic properties can be broadly tailored by controlling the deposition parameters [7,8,9]. In addition, the typically large density of metal nanoclusters in MIFS leads to strong enhancement of the electromagnetic field near the clusters surfaces, enabling other sensing mechanisms such as the amplification of Raman signal [10] or infrared absorption 
[11] of closely located molecules. Thus, MIFs are attractive candidates for the production of performance- and costefficient sensors.

Transmittance measurements are the most straightforward way to optically track changes on the plasmonic response of nanostructures. The spectral shift of transmittance minimum upon variation of surrounding refractive index is often used to quantify the quality of a given nanostructure as sensor, although it has been suggested that monitoring intensity changes may offer larger response [12]. Phase-sensitive measurements such as ellipsometry can offer higher sensitivity than photometric measurements [13, 14], at expense of requiring more demanding instrumental set-up.

It has been recently shown that lossy materials can yield strong interference in films with thickness well below the light wavelength [15], enabling the design and fabrication of ultra-thin optical filters [16] and anti-reflection coatings [17]. In this framework, we propose an alternative sensing scheme that takes profit of the strong dispersion of the optical properties around the surface plasmon resonance of metal-dielectric composites. In particular, we show that reflectance measurements of a system of metal nanoparticles located near a metal surface provides superior sensing performance than transmittance measurements of the same system when placed on a transparent substrate. These improved sensing characteristics are specially advantageous in case of disordered systems such as MIFs.

We start by theoretically investigating the optical response of a composite film consisting of Ag nanoparticles embedded in a dielectric host. Effective optical constants of the composite film are calculated using the MaxwellGarnett theory [18]. The filling fraction of particles, $f$, equals to $1 / 3$ and $\mathrm{Ag}$ optical constants are taken from literature [19]. Figure 1 shows the dependence of the real $\left(n_{e f f}\right)$ and imaginary $\left(k_{e f f}\right)$ part of the composite effective refractive index as a function of the host refractive in$\operatorname{dex}\left(n_{\text {host }}\right)$. The spectral position of the $n_{\text {eff } f}$ peak presents slightly larger variations $(\approx 20 \mathrm{~nm}$ more for the considered $n_{\text {host }}$ values range) than the one of $k_{\text {eff }}$. This observation suggests that optical measurements that have a stronger dependence on $n_{\text {eff } f}$ than on $k_{e f f}$ could present larger sensitivity to detect changes in $n_{\text {host }}$. Thus, the normal incidence transmittance spectra of a $20 \mathrm{~nm}$ thick composite film deposited on a glass substrate (Figure 1.d) is characterized by a minimum whose spectral position closely follows the evolution of $k_{e f f}$ maximum. On the other hand, if the composite film is placed on a metal substrate $(\mathrm{Ag})$, its normal incidence reflectance spectra (Figure 1.c) is characterized by a pronounced dip that appears to be connected with the region of large $n_{\text {ef } f}$ values. It must be mentioned that if a glass substrate is considered, the spectral features are much less remarkable due to the low reflectivity at the film/glass interface, i.e. a highly reflecting substrate is necessary to obtain strong interference effects [20].

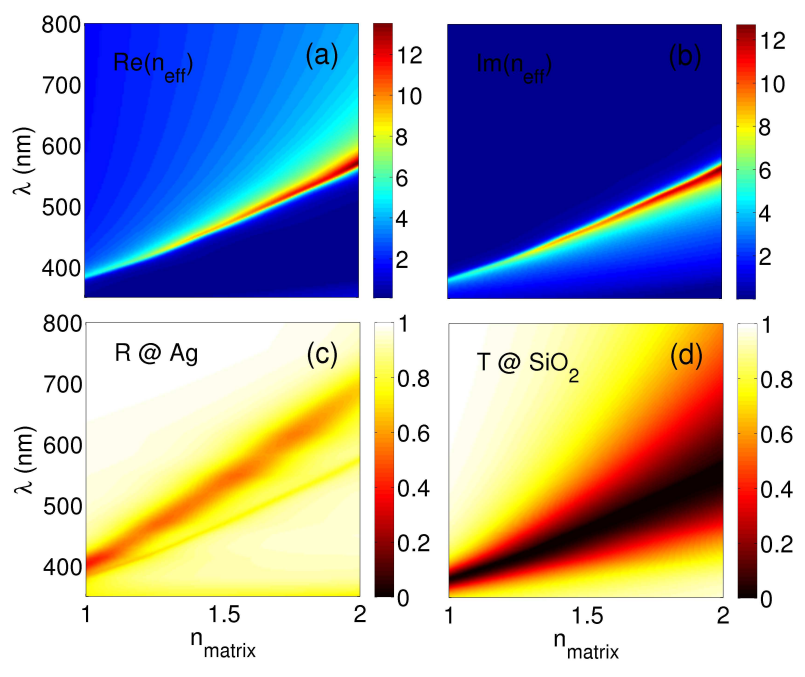

Figure 1 Real (a) and imaginary (b) part of the effective refractive index of an Ag-dielectric composite with $f=1 / 3$ using the Maxwell-Garnett model and corresponding spectra for $20 \mathrm{~nm}$ thick composite films: reflectance on $\mathrm{Ag}$ (c) and transmittance on glass (d) substrates.

In order to understand the observed behaviour we analyze the spectral dependence of different contributions to the overall reflectance, i.e. the first partial wave reflected at the air-composite film interface $\left(r_{1}\right)$ and the multiple partial waves $\left(r_{m}\right)$ reflected at the composite film-substrate interface (Figure 2). At short wavelengths, the composite presents moderate values of $n_{e f f}$ and $k_{e f f}$ and $r_{1}$ is complex while $r_{m}$ is negligible as light is strongly attenuated in the composite film. As the wavelength increases, both $n_{e f f}$ and $k_{e f f}$ increase and $r_{1}$ tends to be purely real and close to -1 , while $r_{m}$ remains small. For larger wavelengths ( $2470 \mathrm{~nm}$ ), $k_{\text {eff }}$ starts to decrease, resulting in nearly real-valued $r_{1}$ and allowing the contribution of $r_{m}$ to grow. From this point on, $r_{m}$ can rapidly vary due to the large values and strong dispersion of $n_{e f f}$ and the nonnegligible values of $k_{e f f}$. In particular, $r_{m}$ can become purely real and positive and cancel out $r_{1}$, giving place to the observed dip in reflectance. At large wavelength values the composite is transparent $\left(k_{e f f} \approx 0\right)$ and the metal substrate becomes close to an ideal perfect electric conductor. Under these conditions the system behaves like a GiresTournois etalon with an overall reflectance close to unity [15].

It has been previously proposed that tracking the reflectance minimum of a sample consisting of metal nanodisks separated from a metal surface by a thin dielectric spacer can be an efficient refractive index sensing scheme in the infrared [21]. The appearance of a reflectance dip has been associated to the excitation of a magnetic-like resonance and the confinement of electromagnetic energy in the spacer layer. In the present case, a similar phenomenol- 


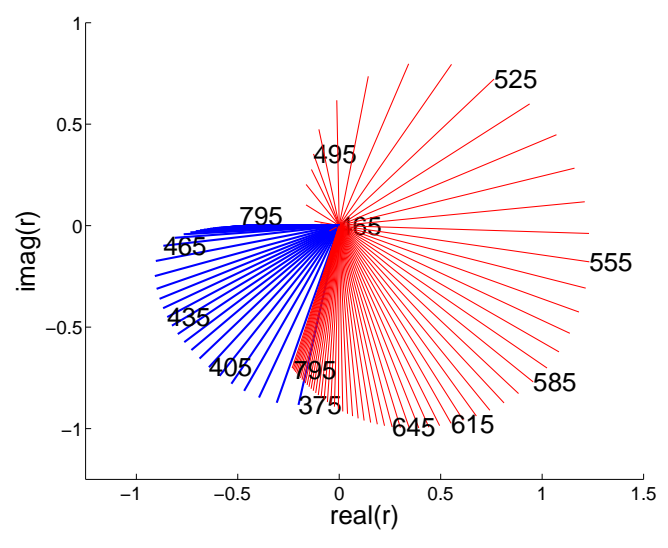

Figure 2 Phasor representation for the contribution to the reflection coefficient for the composite shown in Fig. 1 for $n_{\text {host }}=1.5$. Blue thick lines correspond to contribution from the first reflection at the air-composite interface $\left(r_{1}\right)$ and red thin lines are the contribution from the multiple reflections within the composite film $\left(r_{m}\right)$. Numbers indicate the wavelength of light in $\mathrm{nm}$.

ogy in conceptually close systems can be explained using standard thin film interference notions and effective medium modelling for metal-dielectric composites.

The Maxwell-Garnett model permits to illustrate the key idea of the sensing approach here proposed. However, it is a mean-field theory and neglects local field fluctuations that are particularly important in disordered systems of particles with relatively broad size and shape distributions such as MIFs. In order to obtain a more realistic description of these systems, we perform numerical simulations of the optical response of a disordered arrangement of particles following the Monte Carlo-based procedure proposed by Hinsen and Felderhof [22]. The effective optical constants of an Ag particles-dielectric matrix composite with $f=1 / 3$ as function of $n_{\text {host }}$ are shown in Figure 3. The local field fluctuations give place to surface plasmon resonance broadening [23] in the simulated spectra in comparison to ordered systems, i.e. described by the Maxwell-Garnett model. Numerical simulations reveal that the difference between the spectral position of $n_{\text {ef } f}$ and $k_{\text {eff }}$ peaks is larger $(\approx 150 \mathrm{~nm})$ than in the previous case. As a result, the reflectance spectra of a $20 \mathrm{~nm}$ composite film on Ag substrate (Figure 3.c) shows a dip that is significantly narrower and more rapidly varying with $n_{\text {host }}$ than the dip appearing in the transmittance of the same film when placed on a glass substrate (Figure 3.d).

According to these simulations, it appears that disorder enhances the difference in sensitivity performance between transmittance in transparent substrates and reflectance on metals. This observation is confirmed by computing both the sensitivity (wavelength shift of spectra minimum per change of $n_{\text {host }}$ unit) and figure of merit (sensitivity normalized to the full width at half maximum of the $\operatorname{dip}^{1}$ ) of both sensing schemes, as shown in Table 1. More strikingly, although disordered systems have a broader reflectance dip, the much higher wavelength sensitivity results into a figure of merit that is larger than in ordered systems. This is an unusual observation in plasmonic applications, since disorder mostly spoils or quenches device performance. For instance, disorder significantly reduces the figure of merit for the standard sensing scheme based on tracking transmittance minimum, as shown in the third column of Table 1. The proposed scheme provides sensitivity values that are comparable to sensors based on monodisperse particles with optimized shape and that operate in the visible and near-infrared range [24]. Finally, the reflectance-based sensing scheme also offers large sensing potential for other plasmonic materials: if $\mathrm{Ag}$ is replaced in the composite by more stable $(\mathrm{Au})$ or lower cost $(\mathrm{Cu})$ materials, the advantage of the proposed approach is kept.

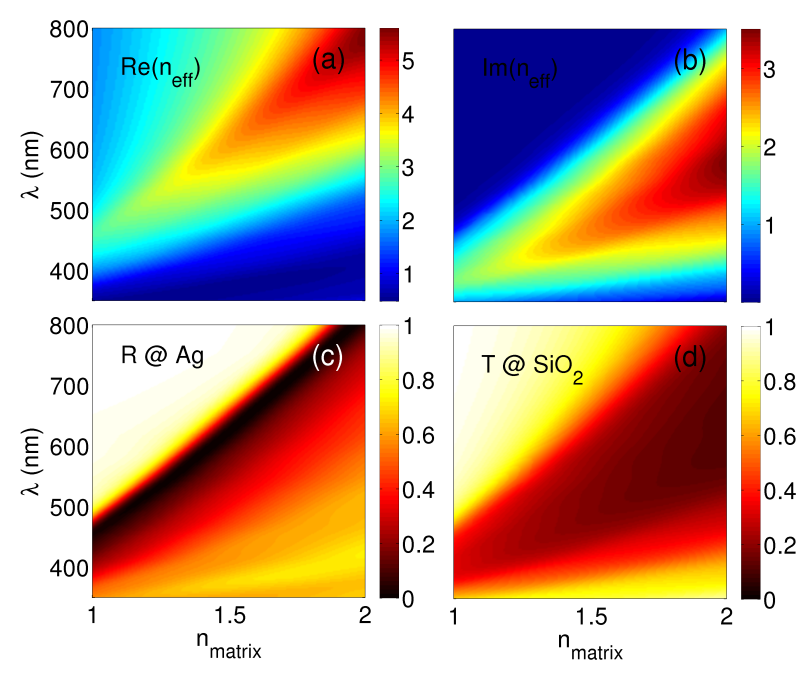

Figure 3 Real (a) and imaginary (b) part of the effective refractive index of an Ag-dielectric composite with $f=1 / 3$ using numerical simulations of disordered systems and corresponding spectra for $20 \mathrm{~nm}$ thick composite films: reflectance on Ag substrate (c) and transmittance on glass (d).

In order to experimentally verify the concepts discussed above, a set of MIFs samples was fabricated using electron beam deposition. The mass thickness of Ag MIFs was set to $7 \mathrm{~nm}$. MIFs were deposited on both bare BK7 substrates and BK7 substrates with pre-deposited $100 \mathrm{~nm}$ thick Ag layer acting as metal substrate and a $3 \mathrm{~nm}$ thick $\mathrm{SiO}_{2}$ film. The small amount of deposited metal results in the formation of an island film made of nanometric particles rather than a compact film. The role of the $3 \mathrm{~nm} \mathrm{SiO} 2$ layer was to provide the same surface for film growth in both types of substrates, which were not pre-heated before

\footnotetext{
${ }^{1}$ Since the spectral dips are inhomogeneous, we choose as full width at half maximum twice the value of the half width taken at the longer part of the spectra
} 
Table 1 Sensitivity $\left(\frac{\Delta \lambda}{\Delta n}\right)$ and figure of merit (FOM) for reflectance $(R)$ and transmittance $(T)$-based schemes for different composites with effective optical constants computed assuming an ordered (Maxwell-Garnett theory, MG) or disordered (Hinsen and Felderhof calculations, HF) arrangement of metal nanoparticles.

\begin{tabular}{ccccc} 
Composite & $\frac{\Delta \lambda}{\Delta n}{ }_{R}(\mathrm{~nm})$ & $\mathrm{FOM}_{R}$ & $\frac{\Delta \lambda}{\Delta n}{ }_{T}(\mathrm{~nm})$ & $\mathrm{FOM}_{T}$ \\
\hline $\mathrm{Ag}(\mathrm{MG})$ & 276 & 3.98 & 178 & 1.12 \\
$\mathrm{Ag}(\mathrm{HF})$ & 381 & 5.15 & 215 & 0.66 \\
\hline $\mathrm{Au}(\mathrm{MG})$ & 223 & 3.57 & 146 & 1.05 \\
$\mathrm{Au}(\mathrm{HF})$ & 324 & 3.64 & 215 & 0.85 \\
\hline $\mathrm{Cu}(\mathrm{MG})$ & 133 & 2.17 & 90 & 0.74 \\
$\mathrm{Cu}(\mathrm{HF})$ & 310 & 3.12 & 176 & 0.71 \\
\hline
\end{tabular}

deposition. Figure 4 shows a plain view scanning electron microcope picture of $\mathrm{Ag}$ islands deposited in this way. The average particle size is $27 \mathrm{~nm}$ with a standard distribution of $8 \mathrm{~nm}$.

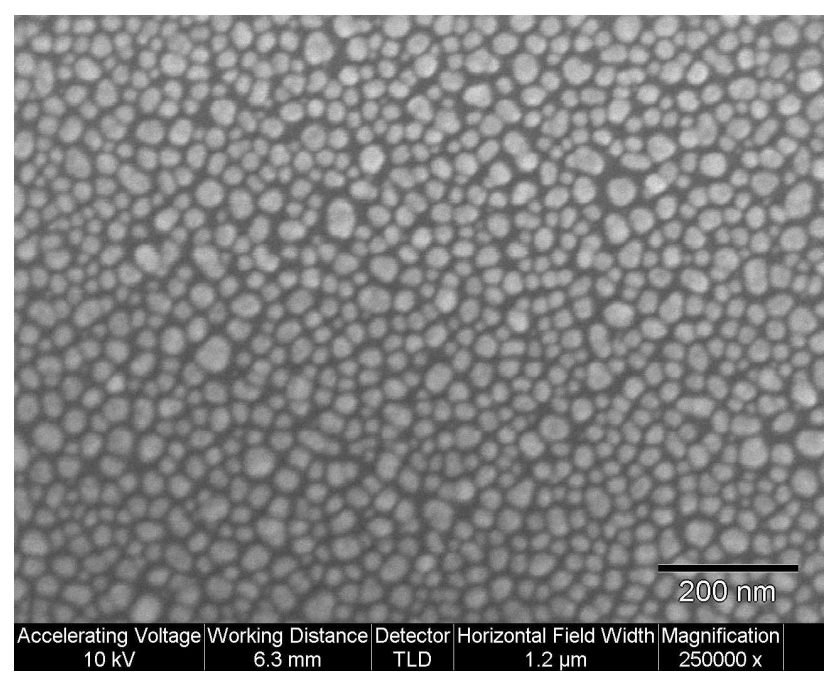

Figure 4 Scanning electron microscopy image of the fabricated uncoated $\mathrm{Ag}$ island film with $7 \mathrm{~nm}$ mass thickness.

In order to check the influence of different dielectric environments, samples were coated with $10 \mathrm{~nm}$ thick $\mathrm{SiO}_{2}$ (refractive index $n \approx 1.5$ ) or $\mathrm{TiO}_{2}(n \approx 2.0)$ films. Figure 5 shows the measured transmittance of the samples deposited on bare BK7 and the reflectance of samples with pre-deposited Ag thick film. In agreement with the theoretical predictions, reflectance of samples on a metal substrate presents a dip that is narrower and more sensitive to $n_{\text {host }}$ than the transmittance dip of samples on transparent substrates. One has to take into account that the presence of a $\mathrm{SiO}_{2}$ or $\mathrm{TiO}_{2}$ film modifies only partially the dielectric environment of the particles, i.e. the experimental shifts are significantly smaller than those shown in Figure 3. Assuming that effective $n_{\text {host }}$ can be roughly approximated as the average of $\mathrm{SiO}_{2}$ and coating layer refractive indices, sensitivity for the transmittance and reflectance-based schemes is quantified as $\approx 110$ and $250 \mathrm{~nm}$ per $n_{\text {host }}$ unit, while the figure of merit is about 4 times larger for the reflectancebased sensor.

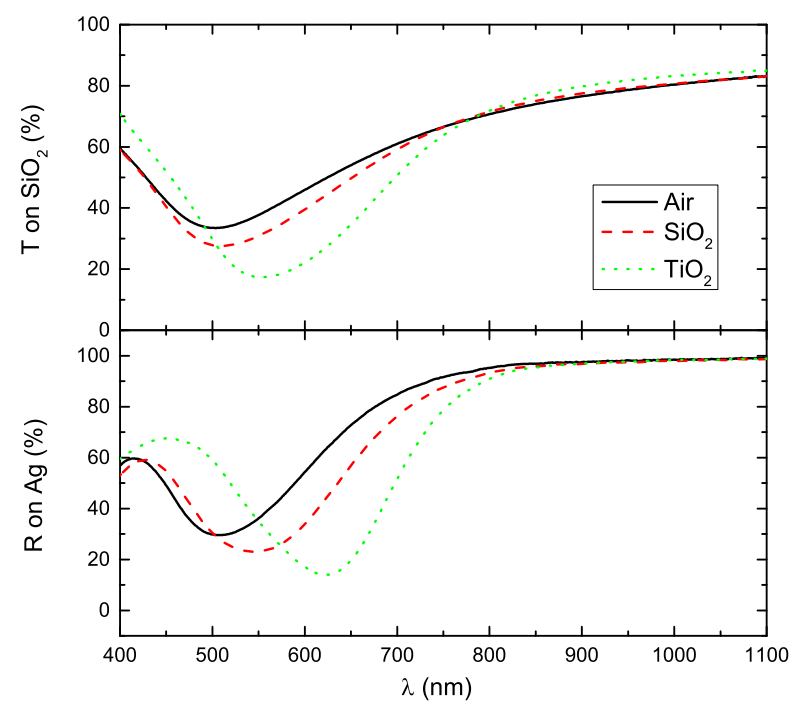

Figure 5 Transmittance on BK7 (up) and reflectance on $\mathrm{Ag}$ (down) spectra of fabricated MIF with $7 \mathrm{~nm}$ mass thickness and covered with different dielectric media.

These figures are in qualitative agreement with those resulting from numerical simulations and it should be taken into account that MIFs possess a more complex geometry than the one considered in calculations. The obtained nanoparticles are not completely spherical but have a certain shape and size distribution and are arranged in a plane, while the numerical simulations assume a monodisperse distribution of spherical particles randomly located in volume. Our previous studies show that these discrepancies prevent an accurate quantitavive comparison between the fabricated samples and the theoretical model, yet the later is able to qualitively account for the influence of randomness and particle concentration [25]. However, the effective medium description is unable to account for a potentially significant effect in the actual samples: the presence of metal nanoparticles close to a metal surface may induce electromagnetic coupling of particles with their images and modify the plasmon resonance [26]. In case of strong coupling, the effective optical behaviour of the actual film can be significantly different from the one predicted for the composite. In order to check the effect of coupling, we fabricate MIFs with a larger amount of metal $(15 \mathrm{~nm}$ mass thickness) and pre-heated the substrates at $120{ }^{\circ} \mathrm{C}$ to enhance island growth and favour spherical shape of the particles [18]. Under these conditions the obtained MIFs are formed by larger particles compared to the deposition without pre-heating: the MIF effective thickness, determined by 
spectroscopic ellipsometry, was above $20 \mathrm{~nm}$ while for the former case was around $10 \mathrm{~nm}$. Figure 6 shows reflectance of MIFs on substrates with pre-deposited Ag thick film and transmittance of MIFs on BK7 substrates upon coating with different dielectric films. The reflectance minimum is significantly red-shifted with respect to transmittance data and the reflectance dip width is now comparable to the one of transmittance. Such different behaviour in comparison with the case of deposition without substrate pre-heating can be explained by the fact that the larger the particle, the stronger its polarizability and hence the strength of image coupling. In addition, polarization parallel to the long axis of the particles results in a weaker image coupling for oblate shapes than for spherical ones [27]. Overall, image coupling induces not only red-shift but also broadening of the spectral dip that partially quenches the performance of the proposed sensing scheme (the sensitivity is slightly larger than in the case of no substrate pre-heating but the figure of merit is more than halved). Therefore, the results suggest that small and oblate nanoparticles are more convenient for sensing purposes.

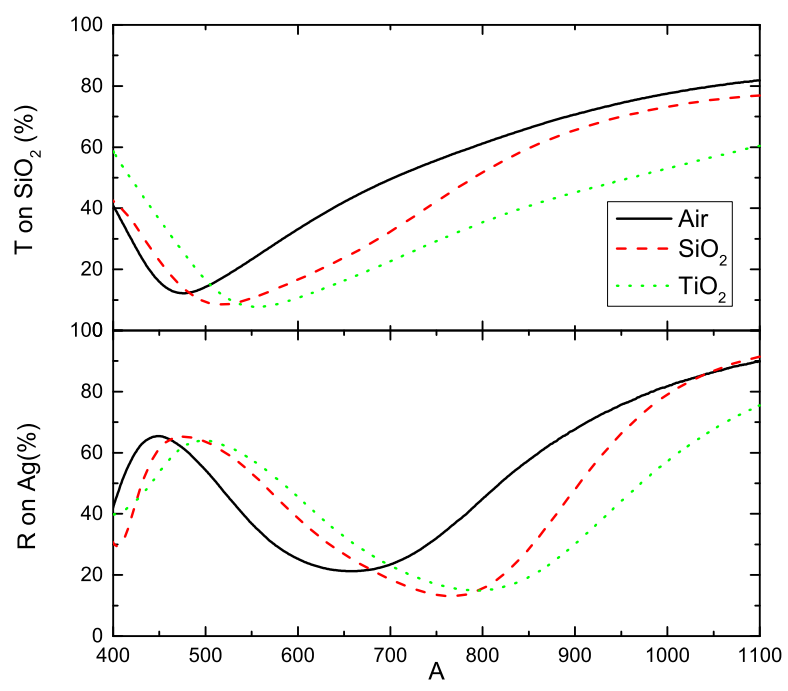

Figure 6 Transmittance on BK7 (up) and reflectance on Ag (down) spectra of fabricated MIF with $15 \mathrm{~nm}$ mass thickness deposited on pre-heated substrates and covered with different dielectric media.

In summary, we present an approach for refractive index sensing based on reflectance measurements of metal island films located close to a metal surface. By exploiting the strong dispersion of optical constants around the surface plasmon resonance, interference effects can take place and provide a way to detect chances in the surrounding of nanoparticles that is more sensitive than standard extinction measurements. The presented scheme is particularly useful in case of disordered arrangement of nanoparticles such as metal island films, that can be easily fabricated us- ing well-established thin film technology and it can help to bridge the gap between potential and practical implementation of plasmonics.

Acknowledgements This work was supported by the Ministry of Environment and Energy, the Ministry of Science and Education, the Environmental Protection and Energy Efficiency Fund and the Croatian Science Foundation under the project "Plasmonic Alternative Materials for Solar Energy Conversion" (PKP-2016-06-4469) in the total amount of 1074000 HRK.

\section{References}

[1] S. A. Maier, Plasmonics: fundamentals and applications (Springer Science \& Business Media, 2007).

[2] J. Homola, Analytical and bioanalytical chemistry 377(3), 528-539 (2003).

[3] A. Vaskevich and I. Rubinstein, Localized Surface Plasmon Resonance (LSPR) Spectroscopy in Biosensing (John Wiley \& Sons, Ltd, 2008).

[4] J. Chen, J. Yuan, Q. Zhang, H. Ge, C. Tang, Y. Liu, and B. Guo, Opt. Mater. Express 8(2), 342-347 (2018).

[5] J. Chen, W. Fan, T. Zhang, C. Tang, X. Chen, J. Wu, D. Li, and Y. Yu, Optics express 25(4), 3675-3681 (2017).

[6] N. Kaiser, Appl. Opt. 41(16), 3053-3060 (2002).

[7] O. Stenzel, The physics of thin-film optical spectra (Springer, 2005).

[8] V. M. Shalaev, Optical properties of nanostructured random media (Springer Science \& Business Media, 2002).

[9] G. Xu, M. Tazawa, P. Jin, S. Nakao, and K. Yoshimura, Applied Physics Letters 82(22), 3811-3813 (2003).

[10] M. Moskovits, Journal of Raman Spectroscopy 36(6-7), 485-496 (2005).

[11] M. Osawa, Surface-enhanced infrared absorption, in: NearField Optics and Surface Plasmon Polaritons, (Springer, 2001), pp. 163-187.

[12] A. B. Dahlin, J. O. Tegenfeldt, and F. Höök, Analytical chemistry 78(13), 4416-4423 (2006).

[13] K. Lodewijks, W. Van Roy, G. Borghs, L. Lagae, and P. Van Dorpe, Nano letters 12(3), 1655-1659 (2012).

[14] V. Janicki, J. Sancho-Parramon, S. Bosch, H. Zorc, F. Belarre, and J. Arbiol, Applied Physics A 115(2), 481-486 (2014).

[15] M. A. Kats, R. Blanchard, S. Ramanathan, and F. Capasso, Optics and Photonics News 25(1), 40-47 (2014).

[16] M. A. Kats, S. J. Byrnes, R. Blanchard, M. Kolle, P. Genevet, J. Aizenberg, and F. Capasso, Applied Physics Letters 103(10), 101104 (2013).

[17] M. K. Hedayati, S. Fahr, C. Etrich, F. Faupel, C. Rockstuhl, and M. Elbahri, Nanoscale 6(11), 6037-6045 (2014).

[18] J. Sancho-Parramon, V. Janicki, and H. Zorc, Optics express 18(26), 26915-26928 (2010).

[19] P. B. Johnson and R. W. Christy, Physical review B 6(12), 4370 (1972)

[20] M. A. Kats, D. Sharma, J. Lin, P. Genevet, R. Blanchard, Z. Yang, M. M. Qazilbash, D. Basov, S. Ramanathan, and F. Capasso, Applied Physics Letters 101(22), 221101 (2012).

[21] N. Liu, M. Mesch, T. Weiss, M. Hentschel, and H. Giessen, Nano letters 10(7), 2342-2348 (2010). 
[22] K. Hinsen and B. Felderhof, Physical Review B 46(20), 12955 (1992).

[23] J. Sancho-Parramon, Nanotechnology 20(23), 235706 (2009).

[24] K. M. Mayer and J.H. Hafner, Chemical reviews 111(6), 3828-3857 (2011).

[25] J. Sancho-Parramon, V. Janicki, and H. Zorc, Optics express 18(26), 26915-26928 (2010).

[26] M. W. Knight, Y. Wu, J. B. Lassiter, P. Nordlander, and N. J. Halas, Nano letters 9(5), 2188-2192 (2009).

[27] C. Noguez, The Journal of Physical Chemistry C 111(10), 3806-3819 (2007)

\section{Graphical Table of Contents}

\section{GTOC image:}

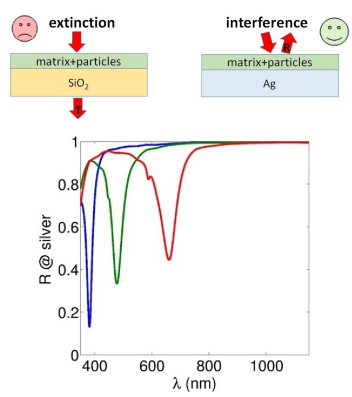

Reflectance measurements of disordered systems of nanoparticles placed in the vicinity of a metal substrate present $\approx 50 \%$ larger sensitivity and several times better figure of merit for refractive index sensing than the extinction-based standard plasmonic sensing schemes. The key element is the use of strong dispersion of optical properties around the surface plasmon resonance to induce high sensitive interference effects. 